\title{
I. V. INFUSION OF MAGNESIUM SULPHATE DURING SPINAL ANAESTHESIA TO IMPROVE POSTOPERATIVE ANALGESIA IN PATIENTS UNDERGOING LOWER EXTREMITY SURGERY.
}



1. Post Graduate Trainee, Department of Anaesthesiology, Mahatma Gandhi Medical College \& Hospital, Jaipur.

2. Post Graduate Trainee, Department of Medicine, Mahatma Gandhi Medical College \& Hospital, Jaipur.

3. Professor, Department of Anaesthesiology, Mahatma Gandhi Medical College \& Hospital, Jaipur.

4. Professor \& Head of the Department, Department of Anaesthesiology, Mahatma Gandhi Medical College \& Hospital, Jaipur.

\section{CORRESPONDING AUTHOR}

Dr. Vandana Tyagi,

B-23, Raghav Marg,

Hanumaan Nagar, Vaishali Nagar,

Jaipur. (Rajasthan) India.

Email-drvandanatyagi@gmail.com

\section{HOW TO CITE THIS ARTICLE:}

Vandana Tyagi, Akash Rajendra, Shashi Chaturvedi, R S Chaudhri. "IV Infusion Magnesium Sulphate during Spinal Anaesthesia to Improve Postoperative Analgesia in Patients Undergoing Lower Extremity Surgery". Journal of Evolution of Medical and Dental Sciences 2013; Vol2, Issue 25, June 24; Page: 4587-4593.

ABSTRACT: BACKGROUND: In a randomized, double-blind, prospective study, we have evaluated the effect of i.v. infusion of magnesium sulphate during spinal anaesthesia, postoperative analgesia and postoperative analgesic requirements in patients undergoing lower extremity surgery. AIM: To evaluate the analgesic efficacy of I.V. infusion of magnesium sulphate during spinal anaesthesia on postoperative pain and analgesic consumption in patients undergoing lower extremity surgery. METHODS AND MATERIAL: Sixty patients undergoing lower extremity surgery under spinal anaesthesia were included. After induction of spinal anaesthesia, the magnesium group (Group M) received intravenous infusion of magnesium sulphate where as in the saline group (Group S) the same volume of isotonic saline over the same period. After surgery, rescue analgesia in form of Inj. Tramadol $1 \mathrm{mg} / \mathrm{kg}$ iv was provided for the patients. The Postoperative pain scores, Rescue analgesic consumption, and incidences of shivering, postoperative nausea, and vomiting evaluated immediately after surgery, and at $30 \mathrm{~min}, 2$, 4, 6, 12, 24 and $48 \mathrm{hr}$ after surgery. Serum magnesium concentrations were checked before the induction of anaesthesia, immediately after surgery, and at $24 \mathrm{~h}$ after surgery. STATISTICAL ANALYSIS: Results were expressed as mean and standard deviation. P value of $<0.05$ was considered statistically significant. RESULTS: Postoperative pain scores were markedly lower in Group $\mathrm{M}$ at 4, 6, 12, 24, and $48 \mathrm{~h}$ after surgery. Cumulative postoperative rescue analgesia consumptions were markedly lower in Group M at 12, 24, and $48 \mathrm{~h}$ after surgery. Postoperative magnesium concentrations were higher in Group M immediately after surgery, but no side-effects associated with hypermagnesemia were observed. Haemodynamic variables and the incidences of shivering, nausea, and vomiting were similar in the two groups. 


\section{ORIGINAL ARTICLE}

CONCLUSION: I.V. magnesium sulphate administration during spinal anaesthesia improves postoperative analgesia without any notable complications.

KEYWORDS: Anaesthetic techniques, subarachnoid; analgesia, rescue analgesia; analgesia, postoperative; ions, magnesium; pain, postoperative.

INTRODUCTION: Spinal anaesthesia provides rapid onset analgesia with good muscle relaxation but relatively lesser post operative pain free period. During postoperative period surgical stress response peaks and has major deleterious effects on all body systems. However a good pre-emptive analgesia attenuates neuro-humoral stress responses to surgery to a great extent. Lower extremity surgery is accompanied by moderate to severe pain after surgery and adequate postoperative pain management is important for early rehabilitation and functional recovery. The goals of pre-emptive analgesia are to decrease post operative pain and the development of chronic pain. It is important to have a pain free postoperative period as it reduces the morbidity and mortality. ${ }^{1}$

Magnesium (Mg) has anti-nociceptive effects due to its antagonistic effect of N-methyl-Daspartate (NMDA) receptor. ${ }^{2}$ NMDA antagonists have been shown to be useful in the reduction of acute postoperative pain, analgesic consumption, or both. Numerous clinical investigations have demonstrated that Mg infusion during GA reduced the anaesthetic requirement and postoperative analgesic consumption ${ }^{3-6}$; whereas other studies suggested that perioperative $\mathrm{Mg}$ administration had little effect on postoperative pain ${ }^{7}$. There are also studies among routes such as intrathecal or epidural that improves anaesthetic and analgesic quality.

However, the effects of systemic Mg during spinal anaesthesia on postoperative analgesia and analgesic consumption have not been fully determined. Accordingly, the aim of this study was to evaluate the effect of an i.v. infusion of magnesium sulphate during spinal anaesthesia on postoperative pain and analgesic consumption along with the haemodynamic changes and adverse effects.

METHODS: This double blind randomized controlled study was done at Mahatma Gandhi Medical College \& Hospital, Jaipur, after permission from hospital ethical committee, for over a period of nine months. 60 patients of ASA grade I \& II were included into two groups comprising 30 patients in each. Exclusion criteria were cardiovascular, hepatic, or renal dysfunction, neuromuscular diseases, opioid or analgesic abuse, and prior treatment with calcium channel blockers. Patients were evaluated prior to surgery and explained about the anaesthetic technique, drugs effect / side effects, use of the visual analogue scale (VAS; 0-no pain; 10-worst possible pain) and perioperative course.

Pre-op., intra-op. and post-op. patient characteristics, hemodynamic parameters and side effects related to drugs was observed and recorded. All patients were given premedication with i.v. midazolam $0.03 \mathrm{mg} / \mathrm{kg}$. Preloading with Lactated Ringer's solution 10ml/kg administered over 15 min and routine non invasive arterial pressure, ECG and pulse oximetry monitoring.

Patients were randomly assigned to a particular group by independent assistant. Spinal anaesthesia performed through the L3-4 or L4-5 interspace and after dural puncture with a 25 G Quincke needle, hyperbaric bupivacaine $0.5 \%$ solution with fentanyl $20 \mu \mathrm{g}$ injected intrathecally. Bupivacaine dose determined on basis of patient's height. (Height $<155 \mathrm{~cm}=12 \mathrm{mg} ; 155-170 \mathrm{~cm}=13 \mathrm{mg} ; 170-$ $180 \mathrm{~cm}=14 \mathrm{mg} ;>180 \mathrm{~cm}=15 \mathrm{mg}$ ). 


\section{ORIGINAL ARTICLE}

After spinal, patients in Group M (Magnesium) had given magnesium sulphate $50 \mathrm{mg} / \mathrm{kg}$ i.v. for $15 \mathrm{~min}$ and then $15 \mathrm{mg} / \mathrm{kg} / \mathrm{hr}$ by continuous i.v. infusion until the end of surgery. Patients in the saline group (Group S) received the same volume of isotonic saline over the same period.

Study data were recorded by an observer unaware of group assignments. The height of spinal block was evaluated for pin-prick sensation $15 \mathrm{~min}$ after intrathecal administration of bupivacaine. If the systolic arterial pressure decreased to below $90 \mathrm{~mm} \mathrm{Hg}$ or if mean arterial pressure decreased $>20 \%$ from baseline, mephentermine $6 \mathrm{mg}$ was given i.v. If heart rate decreased to $<50$ beats $/ \mathrm{min}$, IV atropine $0.6 \mathrm{mg}$ was administered.

Postoperative pain scores were measured using VAS score. During first 4 hrs patients were kept in recovery room and rescue analgesia was provided at VAS $\geq 4$ in form of Inj. Tramadol $1 \mathrm{mg} / \mathrm{kg}$ IV. If VAS score did not decrease to <4, Inj. Tramadol $1 \mathrm{mg} / \mathrm{kg}$ IV repeated with a maximum dose of 3 $\mathrm{mg} / \mathrm{kg}$.

Thereafter, the patients were sent to ward and Inj. Tramadol $1 \mathrm{mg} / \mathrm{kg}$ IV was given on demand. The timing and dosage of rescue analgesic and total consumption of Tramadol during first $48 \mathrm{hrs}$ after operation was noted. Blood samples for serum Mg concentration were obtained before surgery, immediately after \& $24 \mathrm{~h}$ after surgery. Patients' overall satisfaction levels were assessed using a five-point scale (1, very unsatisfactory; 5 , excellent).

The haemodynamic variables, incidences of shivering, postoperative nausea / vomiting, VAS score, total doses of rescue analgesia and all signs / symptoms related to Mg toxicity were evaluated during \& immediately after surgery and at $30 \mathrm{~min}, 2,4,6,12,24$ and $48 \mathrm{hr}$ after the completion of the surgery.

STATISTICS: Results were expressed as mean and standard deviation. The data were compared by applying various tests of statistical analysis as applicable. P value of $<0.05$ was considered statistically significant.

RESULTS: No significant difference was found between the two groups in terms of demographic data like age, gender, weight, ASA grade or duration of surgery (Table 1).

\section{TABLE 1: Demographic Data}

\begin{tabular}{|l|l|l|}
\hline Demographic characteristics & Group M $(\mathrm{n}=30)$ & Group S $(\mathrm{n}=30)$ \\
\hline Age - Mean \pm SD (yrs) & $37.90 \pm 17.81$ & $37 \pm 14.62$ \\
\hline Gender (M/F) & $23 / 7$ & $21 / 9$ \\
\hline Weight(kg) & $60.77 \pm 9.36$ & $61.23 \pm 6.32$ \\
\hline ASA (I/II) & $25 / 5$ & $26 / 4$ \\
\hline Duration of Surgery(min) & $75.97 \pm 22.66$ & $76.43 \pm 20.81$ \\
\hline
\end{tabular}

No technical failure related to spinal anaesthesia occurred and all surgery proceeded without difficulty. The two groups were similar in terms of height of spinal block, mean time to first pain, and the administered dose of bupivacaine (Table 2). 


\section{ORIGINAL ARTICLE}

\begin{tabular}{|l|l|l|}
\hline Spinal characteristics & Group M (n=30) & Group S (n=30) \\
\hline Height of spinal block & T6 (T5-7) & T7 (T5-8) \\
\hline Time to first pain (min) & 245 & 229 \\
\hline Dose of bupivacaine (mg) & 13.2 & 13.4 \\
\hline & & \\
\hline
\end{tabular}

\section{TABLE 2: Spinal Characteristics}

Postoperative serum Mg conc. was higher in Group M than Group S immediately after surgery. However, all patients in Group M had a serum Mg conc. in normal range $24 \mathrm{~h}$ after surgery. Values are presented as mean. Units are as mmol / litre. (Table 3).

\begin{tabular}{|l|l|l|}
\hline Serum Mg concentration & Group M $(\mathrm{n}=30)$ & Group S $(\mathrm{n}=30)$ \\
\hline Preoperative & 0.86 & 0.88 \\
\hline Immediately postoperative & 1.29 & 0.84 \\
\hline Postoperative $24 \mathrm{hr}$ & 0.92 & 0.82 \\
\hline
\end{tabular}

\section{Table 3: Serum Mg Concentration}

There was no significant difference in haemodynamic variables (mean arterial pressure and pulse rate) during the intra- or postoperative period. 2 patients in Group $M$ and 1 patient in Group $S$ experienced bradycardia during surgery, and inj. atropine $0.6 \mathrm{mg}$ was administered. While, 4 patients in Group M and 2 patients in Group S developed hypotension and inj. mephentermine $6 \mathrm{mg}$ administered in each.

Average VAS pain scores were markedly lower in Group M at 4, 6, 12, 24, and $48 \mathrm{~h}$ after surgery. (Figure-1).

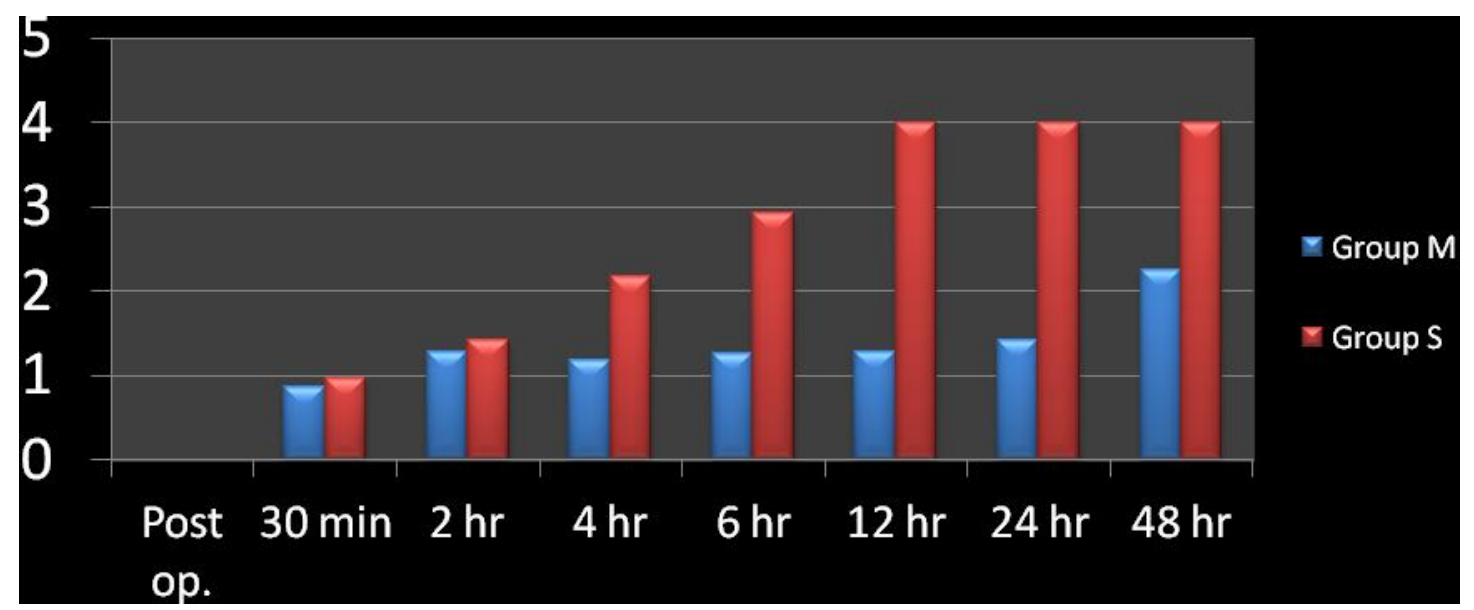

FIGURE 1: VAS PAIN SCORES 
Furthermore, cumulative postoperative rescue analgesia consumptions in form of Inj. Tramadol $1 \mathrm{mg} / \mathrm{kg}$ iv were markedly lower in Group M. (Table 4).

\begin{tabular}{|l|l|l|l|l|}
\hline Rescue Analgesic Time & \multicolumn{2}{|l|}{ Group M (pt.no.) } & \multicolumn{2}{l|}{ Group S (pt. no.) } \\
\hline (Hour) & Hrly no. & cumulative & Hrly no. & cumulative \\
\hline 2 & 0 & 0 & 0 & 0 \\
\hline 4 & 0 & 0 & 0 & 0 \\
\hline 6 & 0 & 0 & 0 & 0 \\
\hline 12 & 0 & 0 & 3 & 3 \\
\hline 24 & 1 & 1 & 9 & 12 \\
\hline 48 & 2 & 3 & 5 & 17 \\
\hline
\end{tabular}

TABLE 4: Rescue Analgesia

The incidences of PONV and shivering after surgery were similar in the two groups. However, global satisfaction scores were significantly higher in Group M. (3.4 v/s 2.6)

DISCUSSION: Regional anaesthesia is preferred to general anaesthesia during the perioperative period and patient controlled epidural analgesia or nerve blocks have also been used in some patients. Improvements in understanding the mechanism of pain have resulted in the administration of analgesics before exposure to pain stimuli to prevent the central sensitization and the amplification of postoperative pain 1 .

Magnesium sulphate has been used in obstetric and cardiac patients. Its use as an adjuvant for perioperative analgesia is based on the properties of NMDA receptor antagonist and calcium channel blocker. However, although the basic mechanism of analgesic effect of $\mathrm{Mg}$ is unclear, it is presumed that its antagonism of NMDA receptor prevents the induction of central sensitization due to peripheral nociceptive stimulation and abolishes hypersensitivity ${ }^{2}$.

Lower extremity surgery is accompanied by moderate to severe pain after surgery and adequate postoperative pain management is important for early rehabilitation and functional recovery. Magnesium sulphate infusion during surgery under spinal anaesthesia reduced postoperative pain and analgesic consumption without any notable complications.

Our observations are in line with the study done by Hwang. et al ${ }^{9}$, where, immediately after spinal block, patients received a $50 \mathrm{mg} / \mathrm{kg}$ bolus of magnesium sulphate followed by a $15 \mathrm{mg} / \mathrm{kg} / \mathrm{hr}$ infusion or saline in the same volumes for $24 \mathrm{~h}$, and postoperative analgesic consumption was significantly lower in the Mg group.

A previous study by Apan et $\mathrm{al}^{8}$ demonstrated that magnesium sulphate infusion started immediately after the induction of spinal anaesthesia and extended for $24 \mathrm{~h}$ reduced analgesic consumption without any effect on spinal block. Here, patients received a $5 \mathrm{mg} / \mathrm{kg}$ bolus of magnesium sulphate followed by a $500 \mathrm{mg} / \mathrm{hr}$ infusion or saline in the same volumes for $24 \mathrm{~h}$, and postoperative analgesic consumption was lower in the Mg group. VAS scores in the two groups during the first $24 \mathrm{~h}$ after surgery was similar except at $12 \mathrm{hr}$ after surgery, and thus, it is assumed that the dosage of magnesium sulphate used was insufficient for postoperative analgesia. However, patients included in that study underwent minor surgical procedures not associated with moderate to severe pain, and postoperative pain scores were similar between the groups other than at $12 \mathrm{~h}$ 


\section{ORIGINAL ARTICLE}

after surgery. While in another study, by Hwang. et $\mathrm{al}^{9}$ a patient-controlled analgesia (PCA) device containing morphine and ketorolac was provided for the patients after surgery.

OUR FINDINGS ARE ALSO IN LINE WITH THE FOLLOWING STUDIES ON I.V. MG: Shashi Kiran et $\mathrm{al}^{4}$ evaluated that single dose of intravenous magnesium sulphate $50 \mathrm{mg} / \mathrm{kg}$ infusion decreases postop pain and requirement of rescue analgesia in patients undergoing inguinal surgery.

Tauzin-Fin $\mathrm{P}$, et $\mathrm{al}{ }^{5}$ conducted a randomized, double-blind, controlled study on males scheduled to undergo radical retropubic prostatectomy. The Mg group received $50 \mathrm{mg} / \mathrm{kg}$ of $\mathrm{MgSO} 4$ and there was decreased postoperative tramadol requirement.

Pre- and intraoperative administration of magnesium sulphate in gynaecology patients receiving total i.v. anaesthesia reduced rocuronium requirement and improved the quality of postoperative analgesia without any significant side-effects ${ }^{6,7}$. Adequate bolus and infusion doses of magnesium sulphate are important for effective analgesia. In a comparison of the effects of three different dose regimens of $\mathrm{Mg}$ on postoperative morphine consumption, a single bolus injection at $40 \mathrm{mg} / \mathrm{kg}$ was found to reduce postoperative morphine consumption, and when this was followed by a maintenance infusion of $10 \mathrm{mg} / \mathrm{kg} / \mathrm{h}$, the effect was enhanced. Moreover, increasing the maintenance infusion to $20 \mathrm{mg} / \mathrm{kg} / \mathrm{h}$ provided no additional advantage and induced unwarranted haemodynamic effects.

Postoperative VAS scores and cumulative rescue analgesic consumption were both markedly lower in Group M at 4, 6 \& $12 \mathrm{hr}$. There was no significant difference of postoperative pain and analgesic use immediately and $30 \mathrm{~min}$ after surgery, attributed to the residual effect of spinal anaesthesia.

After surgery, patients in Group $\mathrm{M}$ had higher serum Mg concentrations than those in Group $\mathrm{S}$, but no side-effects associated with hypermagnesemia. Minor side-effects of parenteral $\mathrm{Mg}$ such as flushing, nausea, and headache are expected at the serum $\mathrm{Mg}$ level above $2 \mathrm{mmol} / \mathrm{litre}$, and potentially life-threatening complications, primarily involved in the cardiovascular and neuromuscular systems, occur when serum Mg concentrations exceed $5 \mathrm{mmol} /$ litre. In the present study, the average serum Mg concentration after surgery in Group M was $1.29 \mathrm{mmol} / \mathrm{litre}$, below the level of minor side-effects.

Magnesium causes a dose-dependent negative inotropic effect, haemodynamic studies have shown that it has a peripheral (predominantly arteriolar) vasodilatory effect ${ }^{12,13}$.

After rapid infusion of 3 or $4 \mathrm{~g}$ of magnesium sulphate, systolic arterial pressure decreased in relation to decreased systemic vascular resistance ${ }^{10}$. In the present study, considering the negative inotropic effect of $\mathrm{Mg}$, prehydration with $500 \mathrm{ml}$ of lactated Ringer's solution was performed and the $\mathrm{Mg}$ bolus dose infused over $15 \mathrm{~min}$. No significant hypotension encountered after administering the Mg bolus dose and also no significant inter-group haemodynamic differences observed during the surgery.

In the present study, time to first pain sensation and spinal block height were similar in the two groups, which is in accord with the findings of a previous study ${ }^{8}$. There was no significant difference between the $\mathrm{Mg}$ and the control groups in terms of spinal block height or the duration of motor block 8,9 .

Magnesium sulphate administration also potentiates neuromuscular block during general anaesthesia ${ }^{6,11}$. It has been shown that the basic mechanisms involve a reduction of the amount of 


\section{ORIGINAL ARTICLE}

acetylcholine released from motor nerve terminals and a decrease in the depolarizing action of acetylcholine at the endplate, or a depression of muscle fibre membrane excitability ${ }^{14}$.

To conclude, intravenous magnesium sulphate infusion given during spinal anaesthesia reduces postoperative pain and analgesic consumption without any complications in the dose schedule. However, further studies are required of different drug dosages of magnesium. Earlier studies with single dose the results are conflicting besides also to establish a dose response relationship with continuous infusion after loading dose.

\section{REFERENCES:}

1. Miller R Erikson L, Feisher L, Wiener-Kronish J, Young W. Miller's Anesthesia. 7th ed. Churchill Livingstone / Elsevier 2009;

2. Robert K Stoelting, Simon C Hillier. Pharmacology \& Physiology in Anesthetic Practice. $4^{\text {th }}$ ed. Lippincott Wiliams \& Wilkins; 618-620.

3. Tramer MR, Schneider J, Marti RA, Rifat K. Role of magnesium sulfate in postoperative analgesia. Anesthesiology 1996; 84: 340-7

4. Shashi Kiran et al. Evaluation of a single-dose of intravenous magnesium sulphate for prevention of postoperative pain after inguinal surgery. Indian Journal of Anaesthesia. 2011 Jan-Feb; 55(1): 31-35

5. Tauzin-Fin $\mathrm{P}$, et al. Intravenous magnesium sulphate decreases postoperative tramadol requirement after radical prostatectomy. European Journal Anaesthesiol. 2006 Dec;23(12):1055-9

6. Ryu JH et al. Effects of magnesium sulphate on intraoperative anaesthetic requirements and postoperative analgesia in gynaecology patients receiving total intravenous anaesthesia. British Journal of Anaesthesia. 2008 Mar; 100(3):397-403.

7. Bhatia Anuj, et al. Effect of intraoperative magnesium infusion on perioperative analgesia in open cholecystectomy. Journal Clinical Anesthesia 2004 Jun; 16(4):262-5.

8. Apan A, Buyukkocak U, Ozcan S, Sari E, Basar H. Postoperative magnesium sulphate infusion reduces analgesic requirements in spinal anaesthesia. European Journal Anaesthesiol 2004; 21: 766-9

9. J.-Y. Hwang. et al. I.V. infusion of magnesium sulphate during spinal anaesthesia improves postoperative analgesia. British Journal of Anaesthesia 104 (1): 89-93 (2010)

10. Dube L, Granry JC. The therapeutic use of magnesium in anesthesiology, intensive care and emergency medicine: a review. Canadian Journal of Anaesthesia 2003; 50: 732-46

11. Seyhan TO, Tugrul M, Sungur MO, et al. Effects of three different dose regimens of magnesium on propofol requirements, haemodynamic variables and postoperative pain relief in gynaecological surgery. British Journal of Anaesthesia 2006; 96: 247-52

12. James MFM. Magnesium: an emerging drug in anaesthesia. British Journal of Anaesthesia 2009; 103: 465-7

13. Ryu J-H, Sohn I-S, Do SH. Controlled hypotension for middle ear surgery: a comparison between remifentanil and magnesium sulphate. British Journal of Anaesthesia 2009; 103: 490-5

14. Ghoneim MM, Long JP. The interaction between magnesium and other neuromuscular blocking agents. Anesthesiology 1970; 32: 23-7 